\title{
Clobazam en Epilepsias Refractarias del Niño
}

\author{
Dra. Dominga Figueroa E. ${ }^{\text {; Dr. León Adjerstein Sch }}{ }^{2}$; Dr. Alejandro Manterola A. ${ }^{3}$

\section{Clobazam in Children with Refractory Epilepsy}

Twenty three patients with refractory epilepsy (14 males and 9 females) and high seizure frecuency, all younger than 18 years old, were treated wilh Clobazam (Frizin $(@$ ) added to conventional anticonvulsants, Doses of $0,5-2$ $\mathrm{mg} / \mathrm{kg} /$ day, not exceding $40 \mathrm{mg} / \mathrm{day}$, were used. Complete seizure reliet was achieved in $46 \%$ of the cases (1l patients) for an average follow up period of 5 months and 27 days. An important reduction in the frecuency of seizures was seen in $21 \%$ of the patients ( 5 cases). Recurrence of seizures after 2 to 3 months of good response to treatment ocurred in $S$ patients $(21 \%)$. The best results were observed in secondary generalized epilepsies.

E1 tratamiento de la epilepsia refractaria aún representa un desafío clínico a pesar de la introducción de nuevas drogas anticonvulsivantes. Partimos de la base que la monoterapia, con control de niveles plasmáticos es el tratamiento de elección, sin embargo en algunos pacientes no se logra buena respuesta con una o más drogas anticonvulsivantes, muchas de las cuales tienen efectos cotaterales potencialmente serios.

1 Médico, Hospital Psiquiátrico "Dr. José Horwitz Barak" Clínica de Psiquintría Infantil.

2 Médicos. Hospital Roberto del Rio. Unidad de Netrrología.

3 Profesor titular de Pediatria. Departamento de Pediatría, División Ciencias Médicas Norte. Facultad de Medicina de la Universidad de Crile.
Una cantidad de estudios publicados en los últimos 20 años han demostrado que la mayoría de las benzodiazepinas poseen propiedades anticonvulsivantes ${ }^{1-2}$. Se ligan con gran afinidad a los sitios receptores del Acido Gama Amino Butírico $(\mathrm{GABA})^{3}$ y ejercen una acción anticon. vulsivante probablemente postsináptica al aumentar la transmisión GABA érgica ${ }^{4}$. érgica 4 .

Clobazam es una 1,5 benzodiazepina, los átomos de nitrógeno están en las posiciones 1 y 5 y un grupo ceto ocupa la posición 4 . El resto de la molécula es similar a la del diazepam. La vida media del clobazam es de 18 irs., pero la del metabolito activo $\mathrm{N}$-desmetil-clobazam es de 36 a 46 hrs. ${ }^{5}$. En 1973 Barzaghi y col. ${ }^{7}$, en experimentos en animales, concluyeron que los 
1,5 derivados eran superiores a los 1,4 en el tratamiento de la epilepsia.

Shenoy en $1982^{8}$ mediante una batería de pruebas anticonvulsivos estandarizados aplicados en ratas, evaluaron las acciones del clobazam, diazepam y dos drogas antiepilépticas no benzodiazepinicas: fenobarbital y valproato. Los resultados obtenidos indican que el clobazam $y$ el valproato muestran una actividad anticonvulsiva experimental más amplia que el fenobarbital y el diazepam.

Durante los últimos 20 años se han hecho estudios clínicos para el tratamiento de la epilepsia crónica con 21 benzodiazepinas ${ }^{9}$. De todas ellas, 3 se han destacado por ser 5 a 10 veces más activas que el diazepam. Ellas son las 1,4 benzo. diazepinas (clorazepam y flunitrazepam [R]) y la 1,5 benzodiazepina (clobazam).

Gastaut $(1978)^{10}$ destacó una respuesta terapéutica rápida a clobazam en el $80 \%$ de los pacientes en todas las formas de epilepsia, tanto sobre las crisis y el trazado EEG como sobre el comportamiento, pero esa respuesta se agotaría parcial o totalmente después de tres semanas de administración en el 33\% de los casos.

Estos mismos resultados obtuvieron Critchley $^{13}$ y Martin ${ }^{14}$ al agregar el clobazam al tratamiento con anticonvulsivantes habituales en pacientes con epilepsias resistentes. Estos autores destacan la ausencia de efectos tóxicos, su mínimo efecto sedante y relajante muscular, su actividad sobre todo tipo de crisis, sus efectos secun. darios favorables con disminución de la ansiedad e inquietud.

Según Gastaut ${ }^{10}$ y Shimizu ${ }^{11}$ el efecto en las epilepsias parciales sería menos regular que en las epilepsias generalizadas secundarias, para las cua. les deberia ser usado como droga de primera línea, especialmente en el síndrome de LennoxGastaut.

Dulac y Figueroa en $1983^{15}$ usaron el clobazam en monoterapia en epilepsias en nifíos, obteniendo una buena respuesta en el $80 \%$ de los casos; las crisis desaparecieron en el $50 \%$ de los pacientes especialmente en epilepsia parcial no lesional.

Con todos estos elementos nos pareció justificado ofrecerle a nuestros pacientes con epilepsia refractaria esta alternativa terapéutica.

\section{MATERIAL Y METODO}

El estudio se realizó en 25 pacientes ambulatorios del Policlinico de Neurología del Hospital Roberto del Río que sufrían epilepsia crónica no controlada, con crisis frecuentes a pesar de recibir hasta 3 drogas anticonvulsivantes en dosis consideradas máxjmas en relación al peso o las concentraciones plasmáticas:

De los 25 pacientes se excluyeron 2 que no volvieron a control. Se controlaron 23 pacientes, 14 hombres y 9 mujeres cuyas edades al comienzo del estudio eran de 1 año 1 mes a 18 años (promedio 8 años 9 meses).

Tabla 1.

Distribución por edad.

lidades

$N^{0}$ de pacientes

$\begin{array}{lr}0 \text { a } 4 \text { años } & 5 \\ 5 \text { a } 9 \text { años } & 7 \\ 10 \text { a } 14 \text { años } & 10 \\ 15 \text { años o más } & 1\end{array}$

Tabla 2.

Frecuencia de las sxisis previa a la administración de clobazam

Frecuencia de crisis

No de pacientes

Más de 20 i día

1 a 20 ; día

1 a $6 /$ semana

2 a $3 /$ mes

5

8

4

Los padres fueron informados y aceptaron colaborar en este nuevo intento terapéutico. Nuestro objetivo fue siempre utilizar el menor número de drogas posible, por lo cual, en todos aquellos pacientes que recibian 3 drogas anticonvulsivantes (9 casos) se suspendió una de ellas al agregar el clobazam (Ravotril $[R]$ en 3 pacientes, primidona en 2, corticoides en 2, fenitoína en 1 y carbamazepina en 1). En los enfermos que recibian 2 drogas (11 casos) se agregó el clobazam y al obtener una respuesta beneficiosa se pudo eliminar una de las drogas previas (carba. mazepina en 4 pacientes, fenitoina en 2, fenobarbital en 1). Sólo 2 pacientes recibían una sola droga, en ambos se habian intentado previamente diversos esquemas terapéuticos sin lograrse e] control de las crisis, en ellos se agregó el clobazam al tratamiento previo.

El diagnóstico del tipo de epilepsia se basó en la historia clínica, examen neurológico, análisis anamnéstico detallado respecto a las características de las crisis y EEG intercrítico. En 5 pacientes se practicó gamaencefalografía o T.A.C.

No se practicaron mediciones plasmáticas de niveles de clobazam: Según la literatura disponible el clobazam no modifica significativamente los niveles plasmáticos de otros anticonvulsivan$\operatorname{tes}^{16}$, por lo cual cualquier modificación en la 
frecuencia de las crisis debiera ser considerada respuesta a la acción específica del clobazam.

En ausencia de una clasificación de las epilepsias adaptada al lactante y al niño nos basamos en la de Masland y clasificamos las epilepsias de la siguiente manera:

Epilepsia parcial no lesional: Pacientes con crisis parciales y EEG focal, sin evidencias clínicas o radiológicas de lesión cerebral focal, con examen neurológico y C.l. normales. Seis pacientes pertenecian a este grupo.

Epilepsin parcial lesional: Pacientes con crisis parciales y EEG focal, con retardo mental y/o signos neurológicos deficitarios atribuibles a una lesión cerebral. Este grupo está formado por 5 pacientes.

Epilepsia generalizada primaria: Corresponde a la epilepsia critogenética o esencial con crisis generalizadas, EEG con descargas bilaterales y sincrónicas y ausencia de sustrato anatómico desmostrable. En este grupo incluimos 2 pacientes con C.l. normal limitrofe, examen neurológico normal, crisis generalizadas tónico-clónicas, ausencias simples y ausencias mioclónicas.

Epilepsia generalizada secundaria: Corresponde a los pacientes en que la enfermedad epiléptica es presumiblemente secundaria a un compromiso orgánico cerebral difuso. Se incluyen 2 pacientes con sindrome de West, 1 con sindrome de Lennox-Gastaut y 7 pacientes que presentaban crisis generalizadas, EEG con descargas generalizadas asincrónicas, multifocales, con signos clinicos de lesión cerebral difusa (retardo mental grave, mictocefalia).

Se administró clobazam 2 veces al día en dosis de $0,5 \mathrm{mg} \times \mathrm{kg} \times$ dia la que se fue aumentando según la respuesta hasta un máximo de $2 \mathrm{mg} \mathrm{x} \mathrm{kg}$ $x$ día $\sin$ sobrepasar los $40 \mathrm{mg} x$ día. Los pacientes fueron controlados una vez por semana. durante el primer mes, más adelante fueron controlados según necesidad por lo menos una vez al mes.

La respuesta al empleo de clobazam se definió como excelente cuando las crisis desaparecieron totalmente; satisfactoria si la frecuencia de las crisis disminuyó a menos del $25 \%$ de la frecuencia inicial; insatisfactoria cuando la frecuencia de las crisis disminuyó pero fue superior al $25 \%$ de la frecuencia previa; Nula cuando no se modificó la frecuencia de las crisis y transitoria en los casos en que después de obtenerse resultados iniciales "excelentes" o "satisfactorios" las crisis volvieron a frecuencias superiores al $25 \%$ de las iniciales al cabo de un tiempo.

\section{RESULTADOS}

Los efectos clínicos anticonvulsivantes se observaron 2 ó 3 días después de comenzar el tratamiento.

Tabla 3.

Resultados según tipo de epilepsia

Exce- Satis- Transi- Insat. lente fact, torio o nulo

$\begin{array}{lrrrr}\text { E. Parcial no lesional } & 5 & - & - & 1 \\ \text { E. Parcial lesionai } & 2 & 1 & 1 & 1 \\ \text { E. Generalizada 1aria } & - & - & 2 & - \\ \text { E. Generalizada 2aria } & 4 & 5 & 2 & - \\ \quad \text { TOTAL } & 11 & 5 & 5 & 2\end{array}$

En las epilepsias parciales no lesionales (6 casos) se obtuvo un control total de las crisis en 5 pacientes obseryados por un período variable desde 3 meses hasta 9 meses (promedio 6 meses). En una paciente la respuesta terapéutica fue nula a pesar de usarse hasta $40 \mathrm{mg} /$ día $(1,2 \mathrm{mg} \mathrm{x} \mathrm{kg} \mathrm{x}$ día), en ella se reemplazó fenitoína por clobazam por presentar importantes reacciones adversas a la fenitoina.

En las epilepsias parciales lesionales ( 5 casos) los resultados fueron variables: en 2 pacientes la respuesta fue excelente; en 1 paciente desaparecieron las crisis los 3 primeros meses para después reaparecer con una frecuencia francamente inferior a la anterior; en otra paciente la respuesta fue satisfactoria y se consideró mejor que la obtenida con ningún otro esquema terapéutico. La respuesta fue insatisfactoria en una paciente con crisis psicomotoras muy frecuentes, en ella no se ha logrado controlar las crisis con ninguna asociación de medicamentos.

En los 2 pacientes con epilepsia generalizada primaria que suftían varios tipos de crisis, la respuesta fue excelente $\operatorname{los} 2$ primeros meses de tratamiento, luego de lo cual reaparecieron las crisis convulsivas. En ellos pudimos observar una mejor acción del clobazam sobre las ausencias que sobre las crisis convulsivas.

En todas las epilepsias generalizadas secunda. rias la respuesta fue excelente o satisfactoria los primeros 2 meses de tratamiento, desgraciadamente en 2 pacientes las crisis reaparecieron al final del segundo mes. En 4 pacientes la respuesta siguió siendo excelente hasta la fecha, 3 tenían más de 10 crisis diarias y su supresión se ma ntuvo durante todo el periodo de observación (mínino 5 meses).

E1 tratamiento con clobazam se mantuvo por un período variable dependiendo de la fecha en que los pacientes ingresaron al estudio y de la 
respuesta obtenida: desde 2 meses hasta 9 meses, 16 pacientes fueron seguidos por más de 5 meses.

Tabla 4.

Relación entre resultados $y$ dosis de clobazam

$\begin{array}{lcccc}\begin{array}{l}\text { Dosis } \\ \text { mg } x \text { kg } x \text { dia }\end{array} & \begin{array}{c}\text { Exce- } \\ \text { lente }\end{array} & \begin{array}{c}\text { Satis- } \\ \text { fact. }\end{array} & \begin{array}{c}\text { Transi- } \\ \text { torio }\end{array} & \begin{array}{c}\text { Insatis- } \\ \text { fact. o } \\ \text { nulo }\end{array} \\ 0,5-0,9 & 2 & - & 1 & - \\ 1-1,4 & 8 & 4 & 2 & 2 \\ 1,5-2 & 1 & 1 & 2 & -\end{array}$

Tabla 5.

Duración del tratamiento y respuesta clínica

$\begin{array}{lcccc}\begin{array}{l}\text { Tiempo de } \\ \text { estudio }\end{array} & \begin{array}{l}\text { Exce- } \\ \text { lente }\end{array} & \begin{array}{l}\text { Satis- } \\ \text { fact. }\end{array} & \begin{array}{c}\text { Transi- } \\ \text { forio }\end{array} & \begin{array}{c}\text { Insatis- } \\ \text { fact, o } \\ \text { nulo }\end{array} \\ \text { - de } 2 \text { meses } & - & - & - & 2 \\ \text { 3 a } 5 \text { meses } & 2 & 1 & 2 & - \\ \text { 5 a } 7 \text { meses } & 6 & 2 & 3 & - \\ \text { + de } 7 \text { meses } & 3 & 2 & - & -\end{array}$

Los 3 pacientes cuya respuesta fue catalogada de transitoria y que fueron seguidos por más de 5 meses, son aquellos en que luego de un periodo sin crisis ellas reaparecieron con una frecuencia correspondiente a la respuesta satisfactoria lo que justificó la mantención del tratamiento.

Se suspendió el clobazam en los 2 pacientes cuya respuesta fue insatisfactoria o nula, ambos recibían $40 \mathrm{mg} /$ día considerada la dosis max́ina.

Efectos indeseables: 7 pacientes ( $30 \%$ ) sufrieron somnolencia al inicio del tratamiento, to que luego de 2 ó 3 días cedió espontáneamente y no volvió a presentarse aún en los casos en que se aumentó la dosis.

En un paciente observamos debilidad muscular que provocó alteración del equilibrio y pseudo ataxia, que cedieron en 2 semanas sin necesidad de reducir la dosis.

Cuatro pacientes presentaron un aumento de la secreción bronquial, todos tenían epilepsia generalizada secundaria, grave retraso psicomotor y daño neurológico. En uno de ellos fue necesario suspender la droga a pesar de una buena respuesta respecto de la frecuencia de las crisis por bronquitis a repetición y bronconeumonia; en otro se suspendió cuando la respuesta se hizo insatisfactoria, en ambos pacientes disminuyeron molestias bronquiales después de suspender el medicamento. No se observaron otros efectos adversos.

Los padres de 14 pacientes (60\%) informaron cambios subjetivos favorables, incluyendo pro- gresos en el desarrollo psicomotor especialmente del lenguaje, mejoría del contacto y el alerta, disminución de la inquietud y de la irritabilidad, etc.

De estos 14 pacientes sólo en 4 se agregó el clobazam al tratamiento previo, en los otros 10 se usó en reemplazo de otra droga.

\section{DISCUSION}

Nuestros resultados son comparables con los obtenidos por otros autores ${ }^{10-14}$

Fueron estimulantes los resultados obtenidos en las epilepsias generalizadas secundarias, ya que la respuesta fue excelente o satisfactoria en 8 pacientes de 10 observados por un periodo promedio de 5 meses 15 días. Esto representa más del doble del número de buenos resultados obte. nidos con otros tipos de anticonvulsivantes y es concordante con los resultados de Gastaut ${ }^{10}$, quiên sugiera que el clobazam sería una droga de primera línea en las epilepsias generalizadas secundarias en las que podría usarse desde el comienzo de la enfermedad.

En las epilepsias parciales los resultados excelentes o satisfactorios se observaron en 8 pacientes de $11(72 \%)$, respuesta similar a las comunicadas con fenobarbital, fenitoina o carbamazepina.

El efecto transitorio en las epilepsias severas ha sido destacado por diversos autores y ocurrió en 5 pacientes $(21 \%)$, todos habían estado sin crisis por más de 2 meses y cuando estas reaparecieron no hubo respuesta favorable al aumento de la dosis. En estos 5 pacientes existían antecedentes de respuesta transitoria con otros anticonvulsivantes lo que sugiere que el fenómeno de "escape" se relaciona también con las características de la enfermedad $y$ no unicamente con algunas particularidades metabólicas del producto administrado.

En ninguno de nuestros pacientes encontramos evidencia de efectos tóxicos parenquimatosos. Gastaut ${ }^{10}$ opina que el clobazam no posee efectos tóxicos ni complicaciones severas como las inducidas por la mayoria de los anticonvulsivantes, por lo que no requeriría control regular de la función hematopoyética, hepática ni renal, hipótesis cuya confirmación excede los objetivos y métodos de nuestro estudio.

\section{RESUMEN}

En un grupo de 23 pacientes ( 14 hombres y 9 mujeres) todos menores de 18 años (edad promedio 8 años 9 meses) con epilepsia refractaria y alta frecuencia de crisis, se indicó clobazam en 
asociación con oțras drogaşanticonvulsivas, 0,5 a $2 \mathrm{mg} \mathrm{x} \mathrm{kg} \mathrm{x}$ dír sin sobrepasar $40 \mathrm{mg} \times$ día.

La actividad inicial del clobazam se hizo evidente en 2 ó 3 días y produjo desaparición total de las ctisis en $47 \%$ de los pacientes (11 casos) por un perioda promedriq de 5 meser 27, dias, y una importante reducción de la frecuencia de las crisis en $21 \%$ de los pacientes ( 5 casos). Observamos un agotamiento del efecto anticonvulsivante luego de 2 o 3 meses de trătàmiento ên 5 pacientes (21\%)

Los resultados obtenidón en las epilepsias generalizadas secundarias fueron notables: en $4 \mathrm{de}$ 10. paejentes las" crisis de aceoierorks durante todo el período de observáción de 50 más mésès. $y$ en otros 4 su frecuencia se redujò a menos del $25 \%$ de la inicial.

\section{REFERENCIAS}

1 Browne, $T_{\text {.; }}$ Pers; $K_{\text {.: }}$ Benzodjazepines in the treatment of epilepsyl; a.review. Epilepsia 14:277, 1993.

2 Kilam, E. L i. Suria, A.: In:" $"$-Benzodiazepine Antiepileptic dtugs. Mechanism of Action". H. Glaser, K. Pẹny and D. Woodbury, Eds. P. 597 Raven Press. Nive Yark, 1980 .

3. Brestrup, $C_{-i}$ Nijelsen, $M$ Benzodiazepines teceptors. Arzaeimittel-Forschung 34:852, 1980.

4 Costa, E.: Benzodiazeprines and neurotransmitlers. Arzneimittel-Forschung 36: 858, 1980.

5 Rupp, W.: Radion, M.; Christ, O.;Hajdu, P.: Pharmacokinetics of single and mintipte doses of clobazam in humans. Britsh Journal of Clinical Pharmacology 7 (Suppi. I) 51. 1979

${ }^{6}$ Barzaghi, Fr Fournex, R.i Maritegazza. P.: Pharma cological and toxicological properties of clobazam.

Arzneimittel-Forschung 23-683, 1973.

${ }^{7}$ Chapman AGi Horton, R.W.; Melarum, B.S.: Antiv convulsivant action of a 1,5 benzodiazepine, clobazam, in reflex epilepsy. Epilepsia 19: 203, 1978.

Shenoy, A.K.; Miyahara, J.T.: Comparations anticonvulsivant activity and neurotoxicidy of clobazam, Diazepam, Phenobarbital and Valproate in Mice abd Rats. Epilepsy 23: 399, 1982

${ }^{9}$ Gastour, H.; Cartier,- J, Dravet, C.: Rger, J.: Mise en evidence par une mothode de screening des proprietes anti-epileptigues exceptionelles d'un benzodiazepine nouvelle. Rev. Neurol. Paris 120: 502, 1969.

10 Gastaut, $H$.. The effects of benzodiazepines on chronic epilepsy in man (with particular reference to ofobazam) Royat Society of Medicine International Congress and Symposium, Series Na,43. Academic Press and The Royal-Society of Medicine (Londres)

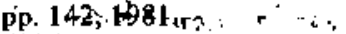

L1 Shimizu, H.; Futrgut $Y:$ Antiepileptic effects of clobazam in children. Brain Dev. 4: 57, 1982.

12 Critchley, E.M.R.; Vakil, S.D.; Hayward, H.W.: Double blind clinical trail of clobazan in refractory epilepsy. Royol Society of Medicine International Congress and Symposium Series No 43. Academic Press and Royal Society of Medicine (Londres) pp. $159,1981$.

13 Manin, A.4.: The antiepileptics effects of clobazam, a long term study in resistant epilepsy Royal Society of Medicine International Congress and Symposium pp. 151,1981 .

14 Duloc, O.; Figueraa, D.; Rey, E.; Arthius, M.: Monotherapie pour le clobazam danş les epilepsies de l'enfant; La Presse Médicale 12: 17, 1983.

15. Vakil. S.D.; Critchkey, E.M.; Cocks, A.: Hayward. H.W.: Royal Society of Medicine International Congress and Symposium. Series No 43, The effect of - clobazam on blood levels of phenobarbitore, phenytoin and carbamazepine (preliminary report) Royal Society of Medicine. Intemational Congress and Symposium Series No 43. Academic Press and Royal Socioty of Medicine (Londres) pp. 165, 1981.

16 Hanks, L.W.: Globazam: Pharmacological and therapeutic profile. Brit. J. Clin. Pharm. (Suppl. 1): $15.15,1979$. 